Review Article

\title{
Expert consensus statement on diagnosis and management of sensitive skin in current clinical practice
}

\author{
Hema Pant ${ }^{1 *}$, Nina Madnani ${ }^{2}$, Sachin Dhawan ${ }^{3}$, Anchala Parthasaradhi ${ }^{4}$, \\ M. K. Shetty ${ }^{5}$, Maya Vedamurthy ${ }^{6}$
}

\begin{abstract}
${ }^{1}$ SCULPT Aesthetic and Cosmetic Clinic, Defence Colony, New Delhi, India
${ }^{2}$ Department of Dermatology, P. D. Hinduja National Hospital and Sir H. N. Reliance Foundation Hospital, Mumbai, Maharashtra, India

${ }^{3}$ Fortis Memorial Research Institute, Skin n Smiles Dermatology and Aesthetics Clinic, Gurugram, Haryana, India

${ }^{4}$ Department of Dermatology Maheswara Medical College, Patancheru, Hyderabad, Telangana, India

${ }^{5}$ Dr. Shetty's Aesthetics, Bangalore, Karnataka, India

${ }^{6}$ RSV Skin and Laser Centre, Chennai, Tamil Nadu, India
\end{abstract}

Received: 17 May 2021

Revised: 22 June 2021

Accepted: 29 June 2021

\author{
*Correspondence: \\ Dr. Hema Pant, \\ E-mail: hemapant.delhi@gmail.com
}

Copyright: ( $)$ the author(s), publisher and licensee Medip Academy. This is an open-access article distributed under the terms of the Creative Commons Attribution Non-Commercial License, which permits unrestricted non-commercial use, distribution, and reproduction in any medium, provided the original work is properly cited.

\begin{abstract}
Sensitive skin is a very commonly seen entity in current dermatological practice that has a significant impact on quality of life. The pathophysiology, classification, diagnosis, and treatment of sensitive skin have evolved over the years, with new studies trying to bring about new robust evidence about the same. The current consensus aims at providing a comprehensive overview on evidence and experience-based approaches for sensitive skin. A total of 35 experts in the field of clinical dermatology participated in expert group meetings organized via teleconference webinar. Current evidence elaborating various aspects like classification, diagnosis, and management of sensitive skin along with clinical experience of experts were discussed in detail. The prerequisites for selecting suitable products, importance of cosmeceuticals as adjunctive therapy and the need for avoiding triggering factors in individuals with sensitive skin as well as associated dermatological disorders were also described. In addition to medical history, physical examination and routine investigations, a draft checklist and its clinical relevance in accurately diagnosing sensitive skin were emphasized upon by the experts. The overall management encompasses avoidance of individual trigger factors, thorough topical therapy using mild, non-irritating ingredients along with adjunctive therapies like moisturizers, sunscreens, and cleansers. The "two-week" strategy was described to be a useful approach for treating sensitive skin. This review article provides a consensus clinical viewpoint of expert dermatologists for effective evaluation, differential diagnosis and appropriate pharmacological as well as non-pharmacological management of sensitive skin in Indian subjects.
\end{abstract}

Keywords: Sensitive skin, Cleanser, Moisturizer, Sunscreen

\section{INTRODUCTION}

Sensitive skin could be described as occurrence of unpleasant sensory responses to stimuli that usually would not incite such sensations. ${ }^{1}$ Sensations like stinging, burning, pain, pruritus, and tingling occur in response to stimuli and cannot be explained by lesions arising due to any skin disease. Sensitive skin is basically a selfdiagnosed entity and is characteristically unaccompanied by any obvious physical signs of irritation. ${ }^{2}$ Environmental 
changes, excessive use of cosmetics, and skin barrier impairment are the major factors responsible for skin hyperreactivity. ${ }^{3}$

Objectively quantifiable signs of irritation are not necessarily seen in individuals with sensitive skin, but subjective sensory effects like itching, burning, stinging, tightness, and dryness are present consistently. ${ }^{1}$

Presence of symptoms, which is almost exclusively subjective, makes diagnosis and subsequent treatment challenging. ${ }^{3}$ In light of the need for in-depth information on various aspects of sensitive skin including epidemiology, triggering factors, pathophysiology, and treatment, this consensus document aims at providing dermatologists' expert opinion on sensitive skin and its effective diagnosis and management in India. Expert group meetings were conducted via teleconference webinar with 36 expert dermatologists across India. Existing evidence and clinical experience with respect to diagnosis of sensitive skin, current treatment modalities and emerging therapies were discussed in detail by the experts. Based on the experts' experience and opinions, several clinical insights were drawn from the advisory boards.

A literature search was performed using databases PubMed and Google Scholar. Relevant articles were identified using keywords like sensitive skin, cleanser, moisturizer, and sunscreen. After screening, 61 suitable articles were identified and included in the paper. This consensus review provides a collation of evidence-based literature on accurate diagnosis and management of sensitive skin. The article has been formulated in accordance with the experts' observations and suggestions.

\section{EPIDEMIOLOGY OF SENSITIVE SKIN}

Surveys have been a widely used approach for evaluating the prevalence of sensitive skin worldwide. ${ }^{1}$ Globally, around $60-70 \%$ of women and $50-60 \%$ of men present with some degree of sensitive skin. ${ }^{1}$ It has been observed that females are significantly more concerned about sensitive skin compared to men. ${ }^{4}$ In India, the average frequency of sensitive skin is $32.3 \%$. Approximately, 400 million Indians might have sensitive or very sensitive skin. $^{5}$

Evidence suggests that young people are more susceptible to sensitive skin. ${ }^{3}$ Tactile sensitivity lessens with age and irritability tests show reduced responses. ${ }^{3}$ Sensitive skin is also more likely to be prevalent in individuals using products like cosmetics, astringents, cleansers, perfumes, and beauty products and in those exposed to hot weather or humidity. ${ }^{1}$

\section{Consensus key point 1}

According to the experts' clinical practice, sensitive skin is a self-diagnosed entity, with around 50-60\% of female patients presenting with it. Women report sensitive skin more frequently than men as they are cosmetically more active, aware, and concerned. The panel also opined that sensitive skin is more commonly seen in the younger age groups (i.e. 20-30 years), due to frequent use of cosmetics and toiletries. Factors like working in hot and humid conditions or misuse of topical steroids also result in sensitive skin.

\section{COMMON LOCATIONS OF SENSITIVE SKIN}

Structural differences in the skin of different body sites may be responsible for variations in barrier function and subsequent variations in skin sensitivity. ${ }^{6}$ Sensitive skin can affect all locations on the body, but it mainly affects the face. ${ }^{6}$ On the face, nasolabial folds are the most sensitive facial region followed by malar eminence, chin, forehead, and upper lip. ${ }^{1}$ Most importantly, the face is exposed to varying environmental conditions on a daily basis and to many products (like cosmetics for women) and practices (like shaving for men). ${ }^{6}$ Sensitive skin may also occur on the scalp, neck, and genital area. ${ }^{1,7}$

\section{Consensus key point 2}

According to the panel experts, sensitive skin is largely seen on the face especially in nasolabial folds, malar areas, and forehead. Other areas include scalp, neck, hands and forearms, genitals, perioral areas, peri-orbital areas, under arms, and cheek areas. The panel further stated that men commonly present with scalp sensitivity, irritation and scaling of scalp, and sensitivity around the beard area. The pediatric population shows a tendency toward periorbital and periocular sensitivity. Patients who wear helmets complain of having sensitivity around the vertex area.

\section{PATHOPHYSIOLOGY OF SENSITIVE SKIN}

Several host-related factors like skin type, gender, and hormonal factors as well as external factors such as climate, exposure to certain products, and cultural influences could lead to self-perceived sensitive skin. ${ }^{1}$ Alterations in skin barrier function and neurosensory dysfunction are two important features which are strongly linked with occurrence of sensitive skin. ${ }^{1}$ It has been hypothesized that sensitive skin may be related to increased permeability of the skin barrier, dry skin, impaired functioning due to skin diseases like atopic dermatitis (AD) and rosacea. ${ }^{8}$ It might also be associated with increased trans-epidermal water loss (TEWL) and reduced quantity of natural moisturizing factor. ${ }^{8}$ Thin stratum corneum, reduced lipids and reduced ceramides have been observed in patients with sensitive skin. ${ }^{1}$ Various hypotheses were proposed to understand the pathophysiology of sensitive skin including role of stratum corneum, transient receptor potential channel, or vasculature. $^{9}$ Moreover, there could be a significant interplay between genetic and environmental factors in the pathogenesis of sensitive skin. ${ }^{8}$ 


\section{Consensus key point 3}

In accordance with the panelists' views, endogenous as well as exogenous factors like genetic predisposition, use of cosmetic products, hormonal changes in females, use of harsh soaps, damage due to exposure to sunlight, noncompliance to prescribed treatment regime as well as individual factors like stress, and past medical history are responsible for sensitive skin.

Dysregulated or impaired stratum corneum (skin barrier) permeability, low levels of skin lipid ceramides, increased TEWL, sweat glands, and epidermal interventions are some of the structural contributors to sensitive skin.

\section{Pons-Guiraud classification}

\section{Very sensitive skin}

Severely reacting to exogenous and endogenous features. The clinical symptoms are acute and permanent; both are associated with psychological reactions.

\section{Environmentally sensitive skin}

Mostly clear, dry and thin skin, mainly reactive to environmental factors like heat and rapid temperature changes, with frequent bouts of flushing

\section{Cosmetically sensitive skin}

Tendency to react to cosmetics. This intolerance is less severe and is mostly limited to some identifiable cosmetic products.
Heightened neurosensory responses may contribute to invisible sensitivity. Increased neurosensory perception may be attributed to more irritable sensory nerves.

\section{CLASSIFICATION OF SENSITIVE SKIN}

Sensitive skin can be described in subjective as well as objective terms. ${ }^{2}$ Subjective perceptions of sensitive skin are seen instantly after cosmetic product application or might be delayed, whereas objective perceptions are based on physician assessment and comprise of the whole range of cutaneous reactions. ${ }^{2}$ Two distinct classifications for sensitive skin known till date are Pons-Guiraud and Muizzuddin classifications (Figure 1). ${ }^{10,11}$

\section{Muizzuddin classification}

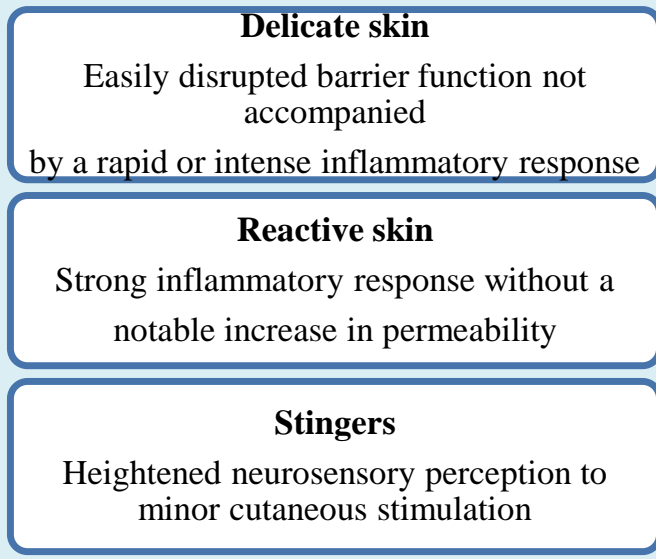

Figure 1: Classification of sensitive skin.

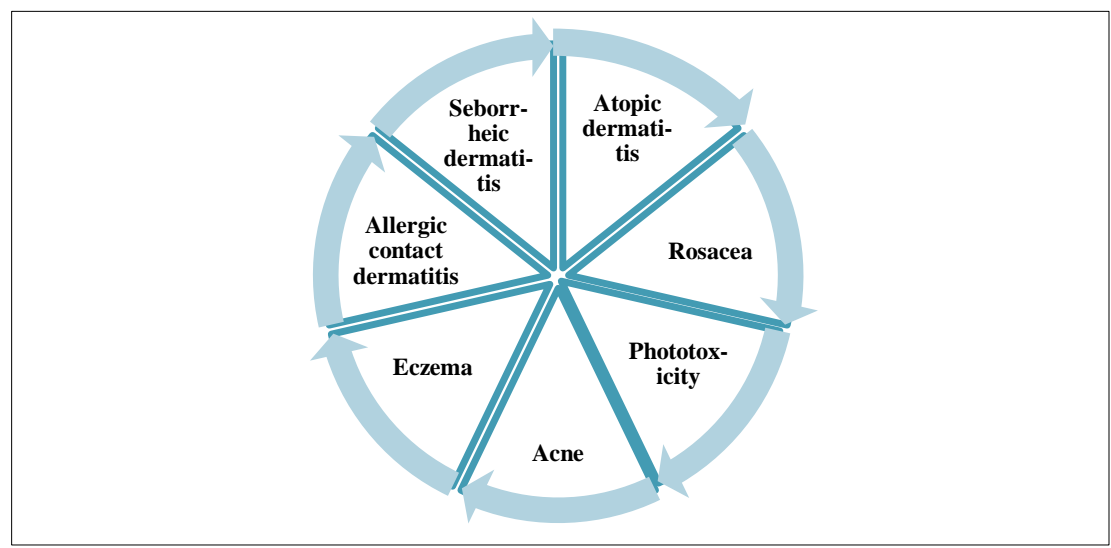

Figure 2: Common differential diagnosis of sensitive skin.

Another classification system is the Mills' and Berger's broad classification, which is not supported by experimental data. ${ }^{12}$ Sensitive skin can also be classified into three types based on epidermal barrier function, namely low barrier function (type I), normal barrier function with inflammatory changes (type II), and normal barrier function without inflammation but with reactivity problems (type III). ${ }^{13}$ However, there is no consensus on 
the proper classification of sensitive skin in literature so far.

\section{Consensus key point 4}

According to the panel experts, Pons-Guiraud classification is used more frequently in current clinical practice. Muizzuddin classification is impractical and difficult to use in daily clinical practice. Also, stingers cannot be identified unless the skin is tested. The possible triggers causing sensitivity can be noted or found out. A comprehensive classification specific for sensitive skin is very difficult.

\section{DIAGNOSIS OF SENSITIVE SKIN}

\section{Medical history and physical examination}

Because sensitive skin is a highly subjective condition, diagnosis should essentially be based on the interpretation of symptoms as reported by the patient and a thorough evaluation of medical history inclusive of personal, family and occupational history, daily habits, and use of cosmetic products. ${ }^{3,14}$ Complete physical examination must also be performed for excluding signs of inflammation and other conditions like contact and atopic dermatitis. ${ }^{3}$ Identifiable clinical parameters like less hydrated and less supple skin, erythema or telangiectasia could be present along with subjective irritation. ${ }^{2}$ Conditions like eczema, AD, rosacea and seborrheic dermatitis are common causes of sensitive skin syndrome (SSS) related to barrier defect, and hence, must be considered during evaluation. ${ }^{4}$

\section{Diagnostic tool for sensitive skin: checklist}

A checklist that includes a series of questions could be a very important diagnostic tool for dermatologists to accurately diagnose SSS. ${ }^{14}$ This list could evaluate patients' self-perception of sensitivity, irritation, and reactivity, and their perception of the reactivity of the skin to cosmetic products and environmental factors. ${ }^{14}$ Table 1 provides important points to be included in a checklist for sensitive skin, as coined by the panel experts.

\section{Clinical tests and investigations}

Various tests are available for assessing neurosensory response, visible cutaneous signs of irritation and for quantifying structural and physiological parameters of the skin on irritant exposure. ${ }^{2}$ A simple patch test would detect any source of allergic contact dermatitis; whereas a photopatch test could be performed in patients with suspected phototoxic contact dermatitis. ${ }^{2,15}$ Also, provocative testing with cosmetics that commonly lead to sensitive skin, or washing tests where patients are asked to wash their face with a particular soap/detergent can be performed. ${ }^{2}$ Dermoscopy and confocal microscopy are other simple diagnostic interventions used for identification of sensitive skin as they help in visualizing structural alterations in the skin. ${ }^{3,14}$
Non-invasive skin assessments, which include tests like TEWL (most widespread), corneometry, colorimetry, squametry and corneosurfametry, have become quite popular. $^{2}$ Furthermore, sensory reactivity tests like stinging test with lactic acid are also used for diagnosing sensitive skin. ${ }^{2}$ In dermatological settings, laboratory tests like blood and urine tests are vital investigations for assessing probable underlying systemic diseases. ${ }^{16}$

\section{Consensus key point 5}

The experts agreed that patient history and visual examination were very important for clinical diagnosis. Diagnosis of sensitive skin should be diagnosis of exclusion because it is a syndrome encompassing various factors. The experts emphasized on the importance of a draft checklist and its clinical relevance in accurately diagnosing sensitive skin. It could enable understanding the patients' perspective of sensitivity and symptoms experienced. Water loss and epidermal integrity should be determined. In addition to the above-mentioned tests, specific blood investigations like complete blood profile, thyroid function test, serum protein levels, and vitamin B12 levels can be done in patients with suspected systemic involvement. In certain patients, depression or psychological aspects may be a factor for aggravation of sensitivity and such patients should be referred to a therapist if required.

\section{Differential diagnosis of sensitive skin}

Dermatologists need to exclude other dermatological conditions for confirming the presence of SSS in patients presenting with erythema. ${ }^{17}$ Presence of erythema can make it difficult to diagnose sensitive skin as compared to various dermatitis conditions. ${ }^{3}$ Skin conditions like atopic dermatitis, acne, rosacea, seborrheic dermatitis, contact dermatitis, psoriasis, and physical urticaria are characterized by altered skin barrier or presence of inflammation. ${ }^{3}$ The frequently seen differential diagnoses are illustrated in Figure 2., ${ }^{3,417}$ Allergic contact dermatitis is a common differential diagnosis of SSS, confirmed by a history of recent contact with a particular substance and/or tracks on the skin. ${ }^{17}$ Rosacea presents with flushing and a feeling of heat, with or without stinging and its course is more transient and persistent than that of SSS. ${ }^{17}$ In absence of erythema, other neuropathic causes linked to small-fiber neuropathies and peripheral neuropathies must be excluded before diagnosing SSS. ${ }^{17}$ Apart from skin conditions, body dysmorphic disorder (BDD), which is a common psychiatric disorder, must always be considered by the dermatologist during evaluation of skin complaints without objective findings. ${ }^{4,18}$

\section{Consensus key point 6}

According to the experts, there is a broad variety of dermatoses, which can mimic SSS. The most common differential diagnosis is AD. Patients can also present with seborrheic dermatitis, acne vulgaris, psoriasis, endogenous 
eczema, contact urticaria, irritant or contact dermatitis, rosacea, drug induced/steroid induced sensitivity, or photosensitivity. BDD can also be associated with sensitive skin.

\section{MANAGEMENT OF SENSITIVE SKIN}

\section{General management approach}

Due to the multifactorial pathogenesis of sensitive skin, several therapies would be needed to control or improve various aspects of the disorder. ${ }^{19}$ The use of moisturizers and optimized lipid mixtures have been seen to improve barrier function. ${ }^{19}$ Moisturizers ideally need to be hydrating, hypoallergenic, non-sensitizing, fragrance-free, non-comedogenic, and cosmetically acceptable. ${ }^{20}$ Products with minimal, pure components and without irritating substances such as urea, alpha-hydroxy acids, propylene glycol, alcohol fragrances, preservatives, and surfactants must be preferred..$^{3,4,8}$ Moreover, emollients like ceramides, squalene, and pseudoceramides that enhance skin hydration, softness, flexibility, and smoothness could be an effective part of routine skin care. ${ }^{20}$ Along with moisturizers, mild cleansers may also have a beneficial role in management of sensitive skin. ${ }^{21}$

Anti-inflammatory compounds like flavonoids and antioxidants reduce inflammatory reactions and modulate vascular reactivity. ${ }^{19}$ Suitable cosmetics and toiletries must be selected for use in patients with sensitive skin. ${ }^{2}$ Soaps must preferably be avoided. ${ }^{4}$ Another vital part of general management includes the use of photoprotectors because ultraviolet radiation could trigger existing symptoms. ${ }^{3}$ Sunscreens (chemical or physical) must be regularly used as a preventive measure for avoidance of hypersensitivity reactions. ${ }^{22,23}$ Topical sunscreens comprise of titanium dioxide, kaolin, talc, zinc oxide, calcium carbonate, and magnesium oxide, which effectively act against ultraviolet rays. ${ }^{22}$ In many sunscreens, antioxidants like vitamin $\mathrm{C}$, vitamin $\mathrm{E}$, silymarin, and green tea polyphenols are added for their protective effects. ${ }^{23}$

Measures like using minimal number of products and protecting the skin from temperature changes must also be implemented. ${ }^{4}$ Moreover, slow re-introduction of the 'minimally necessary' skin care products is indicated whenever needed. ${ }^{4}$ The overall management of sensitive skin can be improved by dermatologists by advising their patients on ways to adjust therapy regimens to best suit their needs and attain optimal outcomes. ${ }^{21}$ Additionally, it is imperative for patients to understand the nature of SSS for balancing expectations and improving adherence to therapy. ${ }^{17}$

\section{Two-week strategy for managing sensitive skin}

The "two-week" strategy could be a useful approach for treating patients with sensitive skin. ${ }^{2}$ This strategy would include the following: discontinuation of all topical cosmetics, over-the-counter (OTC) products, and skin care products and usage of only synthetic detergent (syndet) soap for 2 weeks; discontinuation of all topical prescription medicines like tretinoin, benzoyl peroxide, glycolic acid, or other drying, irritating components for 2 weeks; elimination of all sources of skin friction for 2 weeks; and evaluation of the patient after 2 weeks to investigate if any underlying conditions (like eczema rosacea, or dermatitis.) are present; appropriate treatment of the condition (if present) for at least 2 weeks. ${ }^{24}$

\section{Topical medications for sensitive skin}

Various topical agents are used for treating sensitive skin. In the acute phase, topical calcineurin inhibitors (CNIs) like tacrolimus or pimecrolimus can be prescribed, whereas for long-term management, targeting different mechanisms of SSS with specific treatments is recommended. ${ }^{17}$ Topical CNIs are a safer alternative to topical corticosteroids for treatment of sensitive skin sites. ${ }^{25}$ Nevertheless, application-site irritation, including pain, burning, stinging, and pruritus, has been reported with the use of topical CNIs. ${ }^{25}$ In conditions like eczema and atopic dermatitis, a short-term (2-week) use of corticosteroids could be used to stop the inflammatory process. Prolonged use of topical corticosteroids must be avoided due to risk of steroid-induced SSS. ${ }^{4}$

The aim of using topical formulations in SSS is skin hydration and avoidance of lipid alterations and release of immunomodulators. ${ }^{17}$ Topical formulations with pure substances, without contaminants and low-sensitizing potential preservatives like parabens can be used. ${ }^{17}$ Alkane diols with lower skin irritation potential and surfactants with lower potential for skin irritation must be preferred. ${ }^{17}$

\section{Role of cosmeceuticals in sensitive skin}

In the past few years, cosmeceuticals have been widely accepted in effectively treating sensitive skin, both alone as well as in combination with other treatments. ${ }^{26}$ Polyhydroxy acids (PHAs) like gluconolactone, lactobionic acid, and maltobionic acid as well as $\mathrm{N}$-acetyl glucosamine can support the skin to rebuild dermal density, reinforce barrier function and boost the natural moisturizing factor (NMF) of stratum corneum. ${ }^{26}$ PHAs and bionic acids are basically newer agents with similar benefits as the $\alpha$ - and $\beta$-hydroxy acids, but are devoid of their typical irritation or burning. ${ }^{27}$ Their gentleness on the skin makes them very appropriate for treating sensitive skin. ${ }^{27}$ Also, combinations of PHA and bionic acids in one formulation can rejuvenate and strengthen inflamed skin. ${ }^{26}$

\section{Consensus key point 7}

Apart from the treatments mentioned above, the panel further added that patients should be advised to stop all products containing $\alpha$-hydroxy or $\beta$-hydroxy molecules, lightening products and anti-ageing products. An approach to sensitive skin suggested by the experts was 
'discontinuation of drug, assessment, ascertaining the disease, testing, specific treatment, reintroduction of product and referral (DAATSTRR)'. The panel also stated that patients must be discouraged to self-medicate and use any cosmetic product/home remedy on their own. Implementation of the 2 -week strategy might not be feasible in many cases, especially severe ones; waiting for at least a month is advisable before reintroducing the products. Topical steroids must be avoided as much as possible, specifically when there are no subjective signs. The experts also stated that vitamin D analog-based creams cause irritation of photosensitive skin and hence must not be used for managing sensitive skin. Topical corticosteroids, topical CNIs, or antihistamines could be added in the regimen based on individual patient needs.

\section{Sensitive skin and dermatological conditions}

Sensitive skin shows overlapping symptoms with many dermatological conditions as discussed earlier. In patients with skin disorders like $\mathrm{AD}$, acne, rosacea, and seborrheic dermatitis, sensitive skin is considered as an objective symptom because the condition can present with visible clinical lesions and erythema. ${ }^{3}$ The management for some of the commonly seen disorders is discussed in brief below.

\section{Managing sensitive skin and acne}

Acne is one of the most frequently observed dermatological disorders, and its treatment includes topical and systemic agents. ${ }^{28,29}$ Topical therapy depends on the type and severity of acne. ${ }^{29}$ Mild acne is frequently treated with topical retinoids or numerous agents like azelaic acid, salicylic acid, and benzoyl peroxide (BPO), whereas mild-to-moderate inflammatory acne can be treated with topical anti-inflammatory agents in combination with topical antibiotics. ${ }^{29}$ Topical corticosteroids can be used in severe conditions like very inflammatory acne. ${ }^{29}$ Topical retinoids like adapalene, isotretinoin, tazarotene and tretinoin could be used as monotherapy for inflammatory acne, in combination with more severe forms of acne or as a maintenance treatment. They usually control the formation of microcomedones, decrease formation of lesions and existing comedones, reduce sebum production, normalize desquamation of the epithelium and may also show anti-inflammatory properties. ${ }^{29}$ Lower concentrations of BPO (2.5-5.0\%) and water-based or wash-off agents might be better tolerated in individuals with more sensitive skin. ${ }^{28}$ Furthermore, 20\% azelaic acid can be particularly useful in patients with acne and sensitive skin. ${ }^{28}$

Oral systemic treatment including isotretinoin, oral antibiotics and hormonal agents must be reserved for cases where acne is resistant to topical treatment, in nodular lesions, or in scarring. ${ }^{29}$ Among oral antibiotics used for treating acne, tetracyclines and derivatives are the first choice followed by macrolides, cotrimoxazole, and trimethoprim. ${ }^{30}$ Doxycycline must be avoided due to associated photosensitivity. ${ }^{30}$ Gentle cleansing with lipidfree cleansers, syndets, or other suitable cleansers is important in acne patients. ${ }^{21}$ Non-ionic, fragrance-free dermatologic bars or liquid cleansers with good rinsability could be used..$^{21}$ Non-greasy and water-based moisturizers must also be considered in acne patients for decreasing dryness and stinging associated with barrier disruption, thus enhancing treatment compliance. ${ }^{31}$ The moisturizers and cleansers used in such patients must be noncomedogenic, non-acnegenic, hypoallergenic, and non-irritating to the skin. ${ }^{31}$ Scrubs or other mechanical treatments must be avoided in acne patients with sensitive skin. $^{31}$

\section{Managing sensitive skin and rosacea}

The standard topical therapies for rosacea mainly include metronidazole, azelaic acid, and sulfacetamide-sulfur formulations. $^{32}$ Oral therapy includes certain oral antibiotics like tetracycline, doxycycline, minocycline and less frequently erythromycin, azithromycin, clarithromycin, and clindamycin. ${ }^{32,33}$ Additionally, oral isotretinoin, ivermectin, dapsone, laser therapies, or surgery are also treatment options. ${ }^{33}$ Topical corticosteroids must be avoided. ${ }^{33}$

The skin of patients with rosacea is extremely sensitive to chemical irritants due to which classic soaps, cleansers containing alcohol, astringents, and abrasives must be avoided. ${ }^{21}$ Very mild cleansing agents and moisturizers must be used because rosacea patients already have a compromised skin barrier and sensitive skin. ${ }^{21,32}$ Lipidfree cleansers and syndet cleansers are suitable for rosacea patients. ${ }^{32}$ Therapeutic cleansers with $10 \%$ sulfacetamide and $5 \%$ sulfur in combination with a synthetic detergent are approved for treating rosacea. ${ }^{21}$ General measures like using sunscreens and avoidance of triggers like temperature changes, ultraviolet light, stress, alcohol, and certain foods must also be considered. ${ }^{33}$

\section{Managing sensitive skin and hyperpigmentation}

Sun protection needs to be the primary focus of treating hyperpigmentation. Sunscreens, preferably those containing physical blockers such as titanium dioxide or zinc oxide must be applied regularly on exposed skin. Topical treatment options include retinoids, azelaic acid, hydroquinone, and chemical peels. Hydroquinone is known as the "gold standard" for topical treatment of melasma and hyperpigmentation. Triple fixed-dose combination therapy (fluocinolone acetonide $0.01 \%$, hydroquinone $4 \%$, and tretinoin $0.05 \%$ ) has also become a standard intervention. ${ }^{34}$

Furthermore, cosmeceuticals are commonly used for treating hyperpigmentation with components like kojic acid, arbutin, vitamin $\mathrm{C}$, vitamin $\mathrm{E}$, and niacinamide. Hydroquinone is not preferred as a component of currently available cosmeceuticals due to its unfavorable safety profile. On the other hand, due to lack of side effects, 
various plant extracts like grape seed extract, aloe vera extract, flavonoids, green tea extracts, mulberry extract, and licorice extract are being used in various cosmeceuticals creams. ${ }^{35}$

\section{Managing sensitive skin and seborrheic dermatitis}

Seborrheic dermatitis (SD) is a chronic, recurring, cutaneous condition causing erythema and flaking, occasionally presenting as macules or plaques with dry white or moist oily scales. ${ }^{36}$ Treatment options include topical agents and shampoos containing antifungal agents, anti-inflammatory agents, keratolytic agents, and CNIs. ${ }^{36}$ Antifungal shampoos like 2\% ketoconazole shampoo, $1 \%$ ciclopirox shampoo, and shampoos containing $1.5 \%$ ciclopirox olamine and $1 \%$ zinc pyrithione have shown favorable results in the treatment of SD. ${ }^{36}$ Nonsteroidal treatment options like $1 \%$ pimecrolimus or water-based nonsteroidal topical device creams applied twice-daily are also effectively used. ${ }^{37}$

In cases of persistent SD resistant to topicals, oral antifungals may be preferred. ${ }^{36}$ Evidence suggests that continuous treatment with $200 \mathrm{mg}$ itraconazole daily for 2 days per month maintains the remission of SD with no recurrence for a long time after discontinuation of treatment. ${ }^{38}$ Along with medications, moisturizers with ingredients like biocide piroctone olamine, multiple antioxidants (like telmesteine and tocopheryl acetate) and multiple skin conditioning agents (like ethylhexyl palmitate, bisabolol, and shea butter) could lead to symptomatic improvement. ${ }^{37}$ In cases of facial SD, facial cleansing with gentle soaps twice a day could be beneficial. ${ }^{39}$ Syndet cleanser foams to clean the face in the morning followed by application of non-pharmacological gels/creams are indicated. ${ }^{39}$

\section{Managing sensitive skin and eczema/atopic dermatitis}

The treatment approach for AD must be individualized for the patient according to age, severity and extent of the condition, and distribution of the lesions. ${ }^{40}$ Topical corticosteroids and immunosuppressive agents are the mainstay of treatment together with adjunctive therapeutic moisturizers. ${ }^{41}$ Very potent steroids must be used only in treating AD flares, and as improvement is observed, lowpotency steroids must be used instead with reduced frequency of applications. High-potency steroids ought to be avoided on thin skin, like face or skin folds. Topical CNIs like tacrolimus and pimecrolimus are also used for treating $\mathrm{AD}$ and they show an anti-inflammatory potential similar or slightly less to that of mid-potency corticosteroids. $^{40}$

Basic therapeutic modalities for managing $\mathrm{AD}$ and sensitive skin include effective cleansers that do not compromise skin barrier integrity and moisturizers/emollients that improve skin dryness and restore skin barrier function. ${ }^{41}$ Emollients must ideally contain occlusive agents like lanolin, mineral oils, olive oil, petrolatum ceramide, paraffin, or silicone as they effectively retard transepidermal loss of moisture. ${ }^{42}$ Emollients containing ceramides/pseudoceramides have also been found to positively enhance skin dryness and hydration. ${ }^{42}$ Since individuals with $\mathrm{AD}$ have considerably sensitive skin, avoidance of known irritants, like soaps, chemicals, wool or nylon clothing, abnormal temperature/humidity, or sudden temperature changes is essential. ${ }^{40}$

As soaps have an irritant effect on skin in $\mathrm{AD}$, syndets are an effective option for cleansing due to their mildness and hydrating properties. ${ }^{21,41}$ Lastly, it is vital to protect skin from sun exposure by using broad-spectrum sunscreens. ${ }^{41}$

\section{Managing sensitive scalp}

Sensitive scalp is an extension of sensitive skin and is mainly triggered by pollution, heat, emotional factors, and shampoos. The practices of vigorous herbal hair oil massage or frequent shampooing are predisposing factors, which may worsen the problem in certain individuals and hence must be stopped. Antihistamines and topical steroids like fluticasone lotions could clinically improve sensitive scalp. ${ }^{43}$

\section{Steroid-induced facial sensitivity}

Topical steroid damaged/dependent face (TSDF) is characterized by symptoms occurring due to unsupervised misuse/abuse/overuse of topical steroids on the face over an unspecified and/or prolonged period of time. Common steroids causing this entity include betamethasone valerate, mometasone furoate, clobetasol propionate, and halobetasol propionate. Inappropriate use of steroids was observed in many hyperpigmentation disorders, acne, rosacea, dermatitis, and undiagnosed rashes. ${ }^{44}$

Along with discontinuation of the offending topical steroid, treatment measures include use of broad spectrum sunscreens, emollients, metronidazole, tacrolimus or pimecrolimus, oral tetracyclines, and macrolides. ${ }^{45}$ Management of TSDF necessitates a combined and intensive effort by medical professionals, pharmaceutical companies, and chemists. ${ }^{44}$

\section{Consensus key point 8}

The expert panel agreed to the above-mentioned treatment approaches for sensitive skin and dermatological disorders. They emphasized on the importance of adjunctive therapies like cosmeceuticals and sunscreens in appropriate management. They further added that the use of ketoconazole-based shampoos, sulphate-free shampoos, caffeine-based shampoos, aloe vera based topical sprays, and nutritional supplements could play a role in treating sensitive scalp. They also stated that in case of TSDF, tapering steroid dose rather than abrupt discontinuation is advisable. 


\section{Body dysmorphic disorder}

Prevalence of BDD is commonly seen in dermatology and is most predominant in facial disorders. ${ }^{18} \mathrm{With}$ respect to SSS, dermatologists must know that facial irritation complaints without any distinct findings on examination are a frequent presentation in dysmorphic patients and hence more caution is necessary. ${ }^{4}$ These patients are also at risk of suicidal behavior. ${ }^{4}$

Apart from clinical treatment of skin disorders, evaluating symptoms of BDD by asking patients direct questions on how they feel about their appearance, timely diagnosis, and prompt referral to mental health professionals/services are obligatory for preventing further adverse consequences. ${ }^{418}$ Patients with suspected BDD generally need a detailed diagnostic process for building the trust required for safe referral to mental health professionals. ${ }^{4}$

\section{Consensus key point 9}

According to the experts, patients with BDD have a grossly affected quality of life. Such patients must be screened with the help of questions to check whether their complaints are matching the objective signs indicating sensitive skin. If there is any associated underlying psychological morbidity, a referral is advised.

\section{Role of thermal spring water in sensitive skin}

Thermal spring water (TSW) has been extensively used as a cosmeceutical in the treatment of inflammatory skin diseases for long, but mainly as empirical therapy. The mineral content of TSW is responsible for the skin comfort provided. It is indicated in many chronic inflammatory skin diseases like AD, psoriasis, scars, pruritus, as well as rosacea-like dermatosis and ichthyosis. Moreover, clinical evidence validates the use of selenium-rich TSW as an active ingredient in topical formulations. ${ }^{46}$

\section{Consensus key point 10}

According to the experts, TSW is beneficial, safe, soothing, and economical. It is considered to be a nonirritating, non-inflammatory, and non-pruritic ingredient in moisturizers.

\section{Dermatological procedures and post-procedural care for sensitive skin}

\section{Dermatological procedures}

Ideally, cosmetic procedures must be discontinued or performed sparingly. Potentially irritating procedures like massage, peeling, dermabrasion or roll-off-treatments must be avoided. Over cleansing, use of overdrying/heating masks, and oil treatments must also be avoided. ${ }^{47}$ Chemical peels like mandelic acid peels, salicylic acid peels, and glycolic acid peels have been the most popular non-invasive cosmetic dermatological procedures since long. Superficial peels are frequently used for mild skin disorders like acne, PIH, and melasma. ${ }^{48}$

Microdermabrasion must be used cautiously in patients with sensitive skin or rosacea due to increased risk of irritation and hyperpigmentation. In contrast, microneedling is a safe skin resurfacing therapy and a suitable treatment choice for persons with sensitive skin. ${ }^{49}$

\section{Post-procedural care}

In the immediate post-peel period, a mild soap or non-soap cleanser and calamine lotion in a moisturizing base can be used. Broad spectrum sunscreen ought to be used methodically and sun exposure must be avoided. Patients need to be strictly advised to avoid picking, peeling, scratching or rubbing the skin. ${ }^{50}$

\section{Consensus key point 11}

The experts opined that procedures can be done in patients with sensitive skin after repairing and restoring the skin barrier with proper skin care regimen. Less-invasive procedures must be preferred. Strong peels should be avoided and instead mild peels with kojic acid or azelaic acid must be preferred. Choosing the right concentration and right molecule of the peel is very important in sensitive skin patients. Gel-based peels or mild peels that have less penetration, and less irritation are preferred for shortcontact period. Procedures like microdermabrasion and ablative laser therapies must be avoided. All procedures must be cautiously performed in patients with sensitive skin, keeping in mind the risk of complications and delayed complications. Barrier repair creams like hyaluronic acid-based creams or oatmeal-based ceramide creams are preferred post procedures as they are less occlusive and soothing.

\section{Draft checklist for diagnosing sensitive skin}

\section{Diagnosing sensitive skin checklist}

It included: presence of symptoms like redness, itching, burning, and dryness; family history for skin conditions like atopic dermatitis (AD) and rosacea; reaction to cosmeceuticals or skin care products when applied; irritation due to sun exposure or cold/warm weather; seasonal aggravation and photosensitivity; effect of sudden changes in temperature on skin; relation to menstrual history; relation to stress; sensitivity to particular drugs or dermatological procedures; use of steroids, over-the-counter (OTC) products, or multiple products; history of any exposure to pollution; and frequency of use of home remedies and parlor visits.

\section{CONCLUSION}

Sensitive skin continues to be a challenge in the field of dermatology till date. This consensus article highlights current diagnostic and treatment modalities for sensitive 
skin and associated dermatological disorders. It also highlights the role of adjunctive therapies like moisturizers, sunscreens and cleansers. In spite of having a wide array of therapies for sensitive skin, more robust research is necessary for further efficacious and comprehensive management strategies.

\section{ACKNOWLEDGEMENTS}

We would like to thank Scientimed Solutions Pvt. Ltd. for assisting in manuscript development.

Funding: No funding sources

Conflict of interest: None declared

Ethical approval: Not required

\section{REFERENCES}

1. Farage MA. The prevalence of sensitive skin. Front Med (Lausanne). 2019;6:98.

2. Inamadar AC, Palit A. Sensitive skin: An overview. Indian J Dermatol Venereol Leprol. 2013;79:9-16.

3. Duarte IAG, Silveira JEPS, Hafner MFS, Toyota R, Pedroso DMM. Sensitive skin: review of an ascending concept. An Bras Dermatol. 2017;92:5215.

4. Lev-Tov H, Maibach HI. The sensitive skin syndrome. Indian J Dermatol. 2012;57:419-23.

5. Brenaut E, Misery L, Taieb C. Sensitive skin in the Indian population: An epidemiological approach. Front Med (Lausanne). 2019;6:29.

6. Farage MA. Sensitive skin in the genital area. Front Med (Lausanne). 2019;6:96.

7. Misery L, Ständer S, Szepietowski JC, Reich A, Wallengren J, Evers AW, et al. Definition of sensitive skin: An expert position paper from the special interest group on sensitive skin of the International Forum for the Study of Itch. Acta Derm Venereol. 2017;97:4-6.

8. Misery L, Weisshaar E, Brenaut E, Evers AWM, Huet F, Ständer S, et al. Pathophysiology and management of sensitive skin: position paper from the special interest group on sensitive skin of the International Forum for the Study of Itch (IFSI). J Eur Acad Dermatol Venereol. 2020;34:222-9.

9. Huet F, Misery L. Sensitive skin is a neuropathic disorder. Exp Dermatol. 2019;28:1470-3.

10. Pons-Guiraud A. Sensitive skin: a complex and multifactorial syndrome. J Cosmet Dermatol. 2004;3:145-8.

11. Muizzuddin N, Marenus KD, Maes DH. Factors defining sensitive skin and its treatment. Am J Contact Dermat. 1998;9:170-5.

12. Kligman AM, Sadiq I, Zhen Y, Crosby M. Experimental studies on the nature of sensitive skin. Skin Res Technol. 2006;12:217-22.

13. Yokota T, Matsumoto M, Sakamaki T, Hikima R, Hayashi S, Yanagisawa M, et al. Classification of sensitive skin and development of a treatment system appropriate for each group. IFSCC Mag. 2003;6:3037.

14. Guerra-Tapia A, Serra-Baldrich E, Prieto Cabezas L, González-Guerra E, López-Estebaranz JL. Diagnosis and treatment of sensitive skin syndrome: An algorithm for clinical practice. Actas Dermosifiliogr. 2019;110:800-8.

15. Lazzarini R, Duarte I, Ferreira AL. Patch tests. An Bras Dermatol. 2013;88:879-88.

16. Shin WU, Baek YS, Kim TJ, Oh CH, Kim J. Laboratory tests and compliance of dermatologic outpatients. F1000Res. 2013;2:206.

17. Do LHD, Azizi N, Maibach H. Sensitive skin syndrome: An update. Am J Clin Dermatol. 2020;21:401-9.

18. Akinboro AO, Adelufosi AO, Onayemi O, Asaolu SO. Body dysmorphic disorder in patients attending a dermatology clinic in Nigeria: sociodemographic and clinical correlates. An Bras Dermatol. 2019;94:422-8.

19. Berardesca E, Farage M, Maibach H. Sensitive skin: an overview. Int J Cosmet Sci. 2013;35:2-8.

20. Sethi A, Kaur T, Malhotra SK, Gambhir ML. Moisturizers: The slippery road. Indian J Dermatol. 2016;61:279-87.

21. Mukhopadhyay P. Cleansers and their role in various dermatological disorders. Indian J Dermatol. 2011;56:2-6.

22. Latha MS, Martis J, Shobha V, Sham Shinde R, Bangera S, Krishnankutty B, et al. Sunscreening agents: a review. J Clin Aesthet Dermatol. 2013;6:16-26.

23. Sunscreens and Photoprotection. Available at: https://www.ncbi.nlm.nih.gov/books/NBK537164/. Accessed on 21 August 2020.

24. Draelos ZD. Sensitive skin: perceptions, evaluation, and treatment. Am J Contact Dermat. 1997;8:67-78.

25. Papier A, Strowd LC. Atopic dermatitis: a review of topical nonsteroid therapy. Drugs Context. 2018;7:212521.

26. Bowes L. Using cosmeceutical ingredients and combination regimens for sensitive skin. J Aesth Nurs. 2017;6:522-9.

27. Martin KI, Glaser DA. Cosmeceuticals: the new medicine of beauty. Mo Med. 2011;108:60-3.

28. Zaenglein AL, Pathy AL, Schlosser BJ, Alikhan A, Baldwin HE, Berson DS, et al. Guidelines of care for the management of acne vulgaris. J Am Acad Dermatol. 2016;74:945-73.

29. Fox L, Csongradi C, Aucamp M, du Plessis J, Gerber M. Treatment modalities for acne. Molecules. 2016;21:1063.

30. Rathi SK. Acne vulgaris treatment: the current scenario. Indian J Dermatol. 2011;56:7-13.

31. Goh CL, Noppakun N, Micali G, Azizan NZ, Boonchai W, Chan Y, et al. Meeting the challenges of acne treatment in Asian patients: A review of the role of dermocosmetics as adjunctive therapy. J Cutan Aesthet Surg. 2016;9:85-92. 
32. Levin J, Miller R. A Guide to the ingredients and potential benefits of over-the-counter cleansers and moisturizers for rosacea patients. J Clin Aesthet Dermatol. 2011;4:31-49.

33. Rivero AL, Whitfeld M. An update on the treatment of rosacea. Aust Prescr. 2018;41:20-4.

34. Desai SR. Hyperpigmentation therapy: a review. J Clin Aesthet Dermatol. 2014;7:13-7.

35. Sarkar R, Arora P, Garg KV. Cosmeceuticals for hyperpigmentation: What is available? J Cutan Aesthet Surg. 2013;6:4-11.

36. Gary G. Optimizing treatment approaches in seborrheic dermatitis. J Clin Aesthet Dermatol. 2013;6:44-9.

37. Purnamawati S, Indrastuti N, Danarti R, Saefudin T. The role of moisturizers in addressing various kinds of dermatitis: A review. Clin Med Res. 2017;15:7587.

38. Baysal V, Yildirim M, Ozcanli C, Ceyhan AM. Itraconazole in the treatment of seborrheic dermatitis: a new treatment modality. Int J Dermatol. 2004;43:63-6.

39. Piquero-Casals J, Hexsel D, Mir-Bonafé JF, RozasMuñoz E. Topical non-pharmacological treatment for facial seborrheic dermatitis. Dermatol Ther (Heidelb). 2019;9:469-77.

40. Lee JH, Son SW, Cho SH. A comprehensive review of the treatment of atopic eczema. Allergy Asthma Immunol Res. 2016;8:181-90.

41. Cheong WK. Gentle cleansing and moisturizing for patients with atopic dermatitis and sensitive skin. Am J Clin Dermatol. 2009;10(1):13-7.

42. Hon KL, Kung JSC, Ng WGG, Leung TF. Emollient treatment of atopic dermatitis: latest evidence and clinical considerations. Drugs Context. 2018;7:212530.

43. Godse K, Zawar V. Sensitive scalp. Int J Trichology. 2012;4:102-4.

44. Lahiri K, Coondoo A. Topical steroid damaged/dependent face (TSDF): An entity of cutaneous pharmacodependence. Indian J Dermatol. 2016;61:265-72.

45. Kakkar S, Sharma PK. Topical steroid-dependent face: Response to xylometazoline topical. Indian J Drugs Dermatol. 2017;3:87-9.

46. Seite S. Thermal waters as cosmeceuticals: La Roche-Posay thermal spring water example. Clin Cosmet Investig Dermatol. 2013;6:23-8.

47. Kerscher M, Buntrock H. Treatments for sensitive skin. In: Honari G, Andersen R, Maibach HL, eds. Sensitive skin syndrome. CRC Press. 2017.

48. Castillo DE, Keri JE. Chemical peels in the treatment of acne: patient selection and perspectives. Clin Cosmet Investig Dermatol. 2018;11:365-72.

49. Loesch MM, Somani AK, Kingsley MM, Travers JB, Spandau DF. Skin resurfacing procedures: new and emerging options. Clin Cosmet Investig Dermatol. 2014;7:231-41.

50. Anitha B. Prevention of complications in chemical peeling. J Cutan Aesthet Surg. 2010;3:186-8.

Cite this article as: Pant $\mathrm{H}$, Madnani N, Dhawan S, Parthasaradhi A, Shetty MK, Vedamurthy M. Expert consensus statement on diagnosis and management of sensitive skin in current clinical practice. Int J Res Dermatol 2022;8:132-41. 